$$
\begin{aligned}
& \Phi_{0}=\frac{\lambda v}{\beta}, \quad \Phi_{1}=\frac{1}{2} \frac{\lambda v}{\beta}, \\
& \Phi_{3}=-\frac{1}{8} \frac{\lambda v}{\beta} .
\end{aligned}
$$

b) $v \gg \beta: \Phi_{3}$ ist von der Größenordnung $\beta / v$; im übrigen erhält man:

$$
\begin{aligned}
& \Phi_{0}=\frac{1}{2} \lambda, \quad \Phi_{1}=\frac{1}{3} \lambda, \\
& \Phi_{2}=\frac{1}{8} \lambda .
\end{aligned}
$$

Unsere Berechnungen zeigen, daß in Übereinstimmung mit den schon früher von Bothe erhaltenen Ergebnissen die ungeraden Glieder der Entwicklung (1) nichts zur Aktivität beitragen, wenn die Absorption der Elektronen klein ist. Wenn schon die ersten beiden Glieder der Entwicklung (1) genügen, um das Neutronenfeld zu beschreiben, hängt die Aktivität wegen $\varrho v=K_{0}$ dann nur von der Dichte $\varrho$ und der Geschwindigkeit $v$ der Neutronen ab und nicht von der Orientierung der Sonde. Dasselbe gilt für große Elektronenabsorption verbunden mit kleiner Neutronenabsorption, auch wenn die Anisotropie des Neutronenfeldes stärker ausgeprägt ist. In den übrigen Fällen jedoch bewirkt die Berücksichtigung der Elektronenabsorption, daß die Aktivität von jedem der Glieder in (1) und da- mit auch von der Orientierung der Sonde abhängt, d. h. vom Winkel $\psi$ zwischen Sondennormale und der Verteilungsachse des Neutronenfeldes.

Der anschauliche Grund für dieses Verhalten ist, daß das Zerfallselektron dann eine möglichst große Wahrscheinlichkeit hat, die Sonde wieder zu verlassen, wenn das zugehörige Neutron möglichst nahe an der betrachteten Sondenoberfläche absorbiert wird. Deshalb ist die Aktivität für große $\mu$ dann am größten, wenn der Neutronenstrom senkrecht auf die zu untersuchende Sondenoberfläche zugerichtet ist, und dann am kleinsten, wenn er sie von rückwärts trifft.

Da im allgemeinen die Entwicklung (1) bis $n=1$ notwendig ist, ist die Aktivität wegen $\Phi_{1} \neq 0$ in der Regel nicht nur eine Funktion der Dichte, sondern auch des Neutronenstroms, der auf diese Weise durch die Orientierungsabhängigkeit der Aktivierung der Messung durch die üblichen Sonden zugänglich wird. Wir haben dies durch Messungen zu prüfen versucht, auf die in anderem Zusammenhang eingegangen wird.

Ich danke Herrn Professor Wirtz für die Anregung zu dieser Arbeit und wertvolle Diskussionen. Herrn cand rer. nat. P. von Volckamer danke ich für die Durchführung einer Kontrollberechnung der Koeffizienten (5).

\title{
Zur Thermodynamik der irreversiblen Prozesse III
}

\author{
Von Rolf HaAse
}

Aus dem Institut für Theoretische Hüttenkunde und Physikalische Chemie der Rheinisch-Westfälischen Technischen Hochschule Aachen

(Z. Naturforschg. 8a, 729-740 [1953]; eingegangen am 4. September 1953)

Die Problematik, die in der Formulierung des Ersten Hauptsatzes bei offenen Systemen liegt, wird diskutiert. Es wird gezeigt, daß der Begriff ,Wärmestrom“ nur durch Definition eingeführt werden kann. Die Vorteile bei der Benutzung des sog. ,,reduzierten Wärmestromes" werden auseinandergesetzt.

Die Wärmeleitfähigkeit bei chemisch reagierenden Zweistoffgemischen wird diskutiert, besonders hinsichtlich einer einfachen und übersichtlichen Ableitung der Beziehungen und hinsichtlich einer von Nernst angegebenen Formel für die Wärmeleitfähigkeit eines dissoziierenden idealen Gases.

Die Gültigkeitsgrenzen des Prinzips der minimalen Entropieerzeugung für stationäre Zustände werden untersucht.

$\mathrm{E}$ inige allgemeinere Probleme der Thermodynamik der irreversiblen Prozesse, die in früheren Abhandlungen ${ }^{1,2}$ diskutiert und in einen zusammenfassenden Artikel $^{3}$ übernommen wurden, hängen direkt oder indirekt mit Fragen zusammen, die inzwischen in der Literatur untersucht worden sind. Diese Fragen betreffen
1. das Problem des ,Wärmestromes “ und überhaupt die Formulierung des Ersten Hauptsatzes bei offenen Systemen,

1 R. Haase, Z. Naturforschg. 6a, 420 [1951].

${ }^{2}$ R. Haase, Z. Naturforschg. 6a, 522 [1951].

3 R. Haase, Ergebn. exakt. Naturwiss. 26, 56 [1952]. 
2. die Wärmeleitfähigkeit in chemisch reagierenden Medien,

3. das Prinzip der minimalen Entropieerzeugung bei stationären Nichtgleichgewichtszuständen.

Um die zum Teil etwas verwickelten Situationen zu klären, die durch das Erscheinen mehrerer, voneinander unabhängiger Arbeiten entstanden sind, geben wir im folgenden eine kritische Analyse der drei genannten Probleme.

\section{Zur Formulierung des Ersten Haupt. satzes}

Schon bei der klassischen Formulierung des Ersten Hauptsatzes der Thermodynamik, die nur auf geschlossene Systeme (d. h. Systeme ohne Materieaustausch mit der Umgebung) zugeschnitten ist, stößt man bei genauem Zusehen auf logische Schwierigkeiten, wenn man, der Tradition folgend, den Begriff der „Wärme“ vor den der „Energie“" stellt. Daher hat Born ${ }^{4}$ im Anschluß an Carathéod orys axiomatische Darstellung der Thermodynamik auch den Fragenkomplex des Energiesatzes kritisch analysiert. Von dieser Analyse gehen wir aus, um dann eine entsprechende Untersuchung an offenen Systemen (d. h. Systemen mit Materieaustausch mit der Umgebung) durchzuführen. Wir verzichten auf Definitionen der Begriffe ,Zustand“, ,Zustandsfunktion“, ,Temperatur" usw., da diese in bewährten Darstellungen ${ }^{4,5} \mathrm{zu}$ finden sind. Wir weisen darauf hin, daß diese Begriffe eingeführt werden ohne Vorwegnahme der Definitionen der „,Energie“" und ,Wärme“ bzw. der ,,absoluten Temperatur" und ,Entropie“", die erst durch den Ersten bzw. Zweiten Hauptsatz der Thermodynamik ermöglicht werden.

\section{a) Geschlossene Systeme}

Wir betrachten zunächst ein thermisch isoliertes System. Ein solches System ist dadurch definiert, daß sein Zustand nur dann durch Einflüsse der Umwelt geändert werden kann, wenn am System oder vom System Arbeit geleistet wird. Der Begriff ,,Arbeit" ist hierbei wie in der Mechanik definiert. Ein Prozeß, der in einem thermisch isolierten System abläuft, heißt adiabatisch.

${ }^{4}$ M. Born, Physik Z. 22, 218, 249, 282 [1921]; Natural Philosophy of Cause and Chance, University Press Oxford 1949.
Ein thermisch isoliertes System gehe aus einem Zustand I in einen Zustand II über. Die mechanisch (oder elektrisch) meßbare Arbeit, die während dieser Zustandsänderung am System geleistet wird, sei $A$. Dann lehrt die Erfahrung, daß $A$ für denselben Anfangs- und Endzustand immer denselben Wert hat, gleichgültig, wie die Zustandsänderung erfolgt und welche Arbeitsquellen zur Verfügung stehen. Diese Aussage bildet den Inhalt eines wesentlichen Teils des Ersten Hauptsatzes der Thermodynamik. Sie geht über den Rahmen der Mechanik hinaus, weil z. B. auch eine Änderung der Temperatur eine $\mathrm{Zu}$ standsänderung ist.

Wir können auf Grund der genannten Aussage eine Zustandsfunktion $E$ definieren, deren Zunahme $\Delta E=E_{\mathrm{II}}-E_{\mathrm{I}}$ beim Übergang $\mathrm{I} \rightarrow$ II gegeben ist durch

$$
\Delta E \equiv A
$$

(geschlossenes, thermisch isoliertes System). (1) Man nennt $E$ die Energie des Systems. Demnach kann man den Ersten Hauptsatz der Thermodynamik auch folgendermaßen aussprechen: Die durch Gl. (1) definierte Energie $E$ des Systems ist eine Zustandsfunktion. Daraus folgt dann jede mögliche andere Fassung des Ersten Hauptsatzes, z. B. diejenige über die ,Erhaltung der Energie“ bei abgeschlossenen Systemen und bei Kreisprozessen oder die Aussage, daß bei einer infinitesimalen Zustandsänderung des Systems das Differential d $E$ ein ,, vollständiges Differential" ist.

Es ist wichtig, sich $\mathrm{zu}$ vergegenwärtigen, daß zwei beliebigeZustände I und II eines Systems immer (mindestens in einer Richtung) durch einen adiabatischen Vorgang ineinander überführt werden können, so daß die Energiezunahme $\Delta E$ auch bei einem ganz beliebigen, nicht adiabatisch verlaufenden Prozeß I $\rightarrow$ II gemäß Gl. (1) bestimmt werden kann.

Wir heben nun für unser geschlossenes System die Beschränkung der thermischen Isolierung gegenüber der Umgebung auf. Dann zeigt die Erfahrung, daß bei Temperaturunterschieden zwischen System und Umgebung Zustandsänderungen und damit Energieänderungen im System auftreten können, ohne daß Arbeit geleistet wird ${ }^{5 a}$. Wir sprechen in solchen Fällen von einem ,Wärmeübergang“ oder „Wärmefluß“ zwischen System und Umgebung.

5 E. A. Guggenheim, Thermodynamics, North Holland Publishing Company, Amsterdam 1950.

5a Diese Aussage bildet den zweiten Teil des Ersten Hauptsatzes. 
Die bei einer beliebigen Zustandsänderung I $\rightarrow$ II dem System aus der Umgebung zugeführte Wärme $Q$ wird durch Definition wie folgt festgelegt:

$$
Q \equiv \Delta E-A \text { (geschlossenes System). }
$$

Hierbei ist die Energieänderung $\Delta E$ durch Gl. (1) definiert, und $A$ ist die während der (nicht-adiabatischen) Zustandsänderung am System geleistete Arbeit.

Für eine infinitesimale Zustandsänderung schreiben wir dementsprechend:

$$
\mathrm{d} Q \equiv \mathrm{d} E-\mathrm{d} A \text { (geschlossenes System). }
$$

Hierin ist $\mathrm{d} E$ ein vollständiges Differential, während $\mathrm{d} Q$ und $\mathrm{d} A$ unvollständige Differentiale sind (Wärme $Q$ und Arbeit $A$ sind keine Zustandsfunktionen).

Ist $E_{\text {kin }}$ die makroskopische kinetische Energie des Systems, $E_{\text {pot }}$ die potentielle Energie des Systems in äußeren Kraftfeldern, so findet man, daß die Differenz

$$
E-E_{\text {kin }}-E_{\text {pot }}=U
$$

nur von den inneren Zustandsvariablen des Systems (Temperatur, Druck, Zusammensetzung usw.) abhängt. Man nennt daher $U$ die innere Energie des Systems ${ }^{6}$.

Betrachten wir nun ein homogenes geschlossenes System. Als innere Zustandsvariable wählen wir die Temperatur $T$, den Druck $p$ und die Massen $m_{k}$ $(k=1,2, \ldots, N)$ der einzelnen Stoffe $1,2, \ldots, N$, die im System enthalten sind. Eine infinitesimale Zunahme der Masse $m_{k}$ des Stoffes $k$ bezeichnen wir mit $\mathrm{d}_{\mathrm{i}} m_{k}$, weil hier - im Gegensatz zu den Verhältnissen bei offenen Systemen - Massenänderungen nur durch im Inneren des Systems ablaufende chemische Reaktionen zustande kommen können. Es sei $V$ das Volumen des Systems, $V_{k}$ das partielle spezifische Volumen und $U_{k}$ die partielle spezifische innere Energie des Stoffes $k$. Bei homogenen Systemen interessiert am meisten der Fall, bei dem die am System geleistete Arbeit reversible Kompressionsarbeit und die Änderung der Gesamtenergie gleich derjenigen der inneren Energie ist:

$$
\mathrm{d} A=-p \mathrm{~d} V, \mathrm{~d} E=\mathrm{d} U .
$$

Wir erhalten aus Gl. (3) und (5) für eine infinitesimale Zustandsänderung:

\footnotetext{
${ }^{6}$ Kommt als innere Zustandsvariable nur die Temperatur in Frage, so heißt $U$ auch ,,thermische Energie“. Der vage Begriff ,Wärmeenergie“" sollte ganz vermieden werden.
}

$$
\begin{gathered}
\mathrm{d} Q=\mathrm{d} U+p \mathrm{~d} V \\
\text { (homogenes geschlossenes System) }
\end{gathered}
$$

oder bei Einführen der unabhängigen Variablen $T$,

$$
\begin{aligned}
& p, m_{k}: \\
& \mathrm{d} Q=\frac{\partial U}{\partial T} \mathrm{~d} T+\frac{\partial U}{\partial p} \mathrm{~d} p+\sum_{k=1}^{N} U_{k} \mathrm{~d}_{\mathrm{i}} m_{k} \\
& \quad+p\left(\frac{\partial V}{\partial T} \mathrm{~d} T+\frac{\partial V}{\partial p} \mathrm{~d} p+\sum_{k=1}^{N} V_{k} \mathrm{~d}_{\mathrm{i}} m_{k}\right) .
\end{aligned}
$$

b) Homogene offene Systeme

Die Energie eines offenen Systems zu irgendeinem Zeitpunkt, bezogen auf einen willkürlichen Nullzustand bei der gleichen Masse, läßt sich durch eine sofort einleuchtenđe Verallgemeinerung der Energiedefinition wie folgt festlegen: Man umgebe das System im betrachteten Zeitpunkt mit einer stoffundurchlässigen, thermisch isolierenden Wand und bestimme die Arbeit $A$, die notwendig ist, um das System aus dem willkürlich gewählten Bezugszustand I in den betrachteten Zustand II zu bringen. Die Energiedifferenz $\Delta E=E_{\mathrm{II}}-E_{\mathrm{I}}$ ist dann gemäß Gl. (1) durch $\Delta E=A$ gegeben.

Die einem offenen System aus der Umgebung zugeführte ,Wärme“ ist nicht mehr so eindeutig definierbar wie die Energie eines solchen Systems ${ }^{6}$. In der Tat benutzen verschiedene Autoren verschiedene Wärmedefinitionen. Auf diesem Umstand beruhen zum großen Teil die Schwierigkeiten, auf die man beim Vergleich der Rechnungen mehrerer Forscher auf dem Gebiet der Thermodynamik der irreversiblen Prozesse stößt. Wir werden aus den vielen möglichen Definitionen eine auswählen, die sich nachträglich als die zweckmäßigste erweisen wird.

Der Übersichtlichkeit halber betrachten wir ein homogenes offenes System. Wir wählen als innere Zustandsvariable wiederum $T, p$ und $m_{k}(k=1,2$, $\ldots, N)$, bedenken aber, daß jetzt für eine infinitesimale Massenänderung gilt:

$$
\mathrm{d} m_{k}=\mathrm{d}_{\mathrm{i}} m_{k}+\mathrm{d}_{\mathrm{a}} m_{k},
$$

wobei $\mathrm{d}_{\mathrm{a}} m_{k}$ die infinitesimale Zunahme der Masse des Stoffes $k$ durch Zufuhr von außen ist.

Wegen Gl. (8) sind für homogene offene Systeme , lie Gln. (6) und (7) nicht mehr identisch.Wir wählen Gl. (7) als Definition der einem homogenen offenen

\footnotetext{
6a Dasselbe gilt auch für die an einem offenen $\mathrm{Sy}$ stem geleistete „Arbeit“. Vgl. R. Defay, Bull. Acad. roy. Belgique (Cl, Sc.) 15, 678 [1929].
} 
System aus der Umgebung zugeführten (infinitesimalen) Wärme. Beachten wir die Beziehungen?

$\mathrm{d} U=\frac{\partial U}{\partial T} \mathrm{~d} T+\frac{\partial U}{\partial p} \mathrm{~d} p+\sum_{k=1}^{N} U_{k} \mathrm{~d} m_{k}$,

$\mathrm{d} V=\frac{\partial \boldsymbol{V}}{\partial T} \mathrm{~d} T+\frac{\partial \boldsymbol{V}}{\partial p} \mathrm{~d} p+\sum_{k=1}^{N} V_{k} \mathrm{~d} m_{k}$,

so finden wir mit Gl. (8), daß die Definition (7) auch folgendermaßen formuliert werden kann:

$\mathrm{d} Q \equiv \mathrm{d} U-\sum_{k=1}^{N} U_{k} \mathrm{~d}_{\mathrm{a}} m_{k}+p \mathrm{~d} V-p \sum_{k=1}^{N} V_{k} \mathrm{~d}_{\mathrm{a}} m_{k}$

(homogenes offenes System)

oder nach Einführen der partiellen spezifischen Enthalpien $H_{k}=U_{k}+p V_{k}$ :

$$
\mathrm{d} Q \equiv \mathrm{d} U+p \mathrm{~d} V-\sum_{k=1}^{N} H_{k} \mathrm{~d}_{\mathrm{a}} m_{k}
$$

(homogenes offenes System).

Für ein homogenes geschlossenes System $\left(\mathrm{d}_{\mathrm{a}} m_{k}=0\right.$, $k=1,2, \ldots, N)$ erhalten wir Gl. (6) zurück.

Die Definitionsgleichung (11) entspricht einer von Gillespie und $\mathrm{Coe}^{8}$ benutzten Beziehung8a. Die Größe $\mathrm{d} Q / \mathrm{d} t$ ( $t=$ Zeit) ist der Wärmestrom aus der Umgebung und wurde bisher vom Verfasser ${ }^{1,3}$ als „,reduzierter Wärmestrom" $\left(\mathrm{d}_{\mathrm{z}} Q / \mathrm{d} t\right)$ bezeichnet.

Die sonst noch in der Literatur (siehe z. B. ${ }^{9,10}$ ) als ,Wärme“ bezeichneten Größen $Q^{\prime}$ sind von der allgemeinen Gestalt

$$
\mathrm{d} Q^{\prime}=\mathrm{d} Q+\sum_{k=1}^{N} \gamma_{k} \mathrm{~d}_{\mathrm{a}} m_{k}
$$

wobei die $\gamma_{k}$ irgendwelche Zustandsgrößen sind.

\section{c) Diskontinuierliche Systeme}

Wir betrachten den einfachsten Fall eines diskontinuierlichen Systemes: zwei homogene offene Systeme (,Phasen“), gekennzeichnet durch die Indices ' und ", die durch eine Phasengrenzfläche, eine Membran oder dergleichen voneinander getrennt sind und zwischen denen ein Stoff- und Ener-

${ }^{7}$ Die Gln. (9a), (9 b) sind deshalb sowohl für geschlossene als auch für offene homogene Systeme gültig, weil nur Differentiale von Zustandsvariablen darin vorkommen. Aus dem gleichen Grunde gilt auch die Gibbs sche Fundamentalgleichung für jede (reversible oder irreversible) Zustandsänderung einer beliebigen (geschlossenen oder offenen) Phase, wenn deren innerer Zustand vollständig durch die Variablen $T, p, m_{k}$ beschrieben werden kann.

${ }^{8}$ L. J. Gillespie u. J. R. Coe jr., J. chem. Physics 1, 103 [1933]. gieaustausch stattfinden kann. (Von chemischen Reaktionen, elektrischen Potentialdifferenzen, Grenzflächenerscheinungen usw. sehen wir ab.) Die Phase ' bzw." sei von einem ,Kalorimeter" (* bzw. **) umgeben, d. h. von einem Medium, das nur mit der betreffenden Phase in Energieaustausch steht und bei dem wir die $\mathrm{Zu}$ - oder Abnahme der inneren Energie $U^{*}$ bzw. $U^{* *}$ durch Messung von Temperaturänderungen feststellen können. Da ein Kalorimeter ein geschlossenes System ist, an dem (praktisch) keine Arbeit geleistet wird, gilt gemäß Gl. (3) und (5) für infinitesimale Änderungen:

$$
\begin{gathered}
\mathrm{d}_{\mathrm{a}}{ }^{\prime} Q=-\mathrm{d} U^{*}, \\
\mathrm{~d}_{\mathrm{a}}{ }^{\prime \prime} Q=-\mathrm{d} U^{* *},
\end{gathered}
$$

worin $\mathrm{d}_{\mathrm{a}}{ }^{\prime} Q$ bzw. $\mathrm{d}_{\mathrm{a}}{ }^{\prime \prime} Q$ die dem offenen System' bzw. " von ,außen", d. h. aus dem Kalorimeter * bzw. ** zugeführte infinitesimale Wärme ist. Gemäß Gl. (13) sind $\mathrm{d}_{\mathrm{a}}{ }^{\prime} Q$ und $\mathrm{d}_{\mathrm{a}}{ }^{\prime \prime} Q$ meßbare Größen.

Da das gesamte System, bestehend aus den beiden Phasen und den beiden Kalorimetern, geschlossen und thermisch isoliert ist, folgt aus Gl. (1), (5) und (13):

$\mathrm{d} U^{\prime}+p^{\prime} \mathrm{d} V^{\prime}+\mathrm{d} U^{\prime \prime}+p^{\prime \prime} \mathrm{d} V^{\prime \prime}=\mathrm{d}_{\mathrm{a}}{ }^{\prime} Q+\mathrm{d}_{\mathrm{a}}{ }^{\prime \prime} Q$,

wobei wir die Eigenschaft der Additivität der Energien der Teilsysteme benutzt haben. Wir definieren nun in folgerichtiger Verallgemeinerung von Gl. (11) als die der Phase ' bzw. " von ,,innen“, d. h. jeweils von der anderen Phase zugeführte infinitesimale Wärme $\mathrm{d}_{\mathrm{i}}{ }^{\prime} Q$ bzw. $\mathrm{d}_{\mathrm{i}}{ }^{\prime \prime} Q$ :

$$
\begin{aligned}
& \mathrm{d}_{\mathrm{i}}{ }^{2} Q \equiv \mathrm{d} U^{\prime}+p^{\prime} \mathrm{d} V^{\prime}-\sum_{k} H_{k}{ }^{\prime} \mathrm{d} m_{k}{ }^{\prime}-\mathrm{d}_{\mathrm{a}}{ }^{\prime} Q, \\
& \mathrm{~d}_{\mathrm{i}}{ }^{\prime \prime} Q \equiv \mathrm{d} U^{\prime \prime}+p^{\prime \prime} \mathrm{d} V^{\prime \prime}-\sum_{k} H_{k}{ }^{\prime \prime} \mathrm{d} m_{k}{ }^{\prime \prime}-\mathrm{d}_{\mathrm{a}}{ }^{\prime \prime} Q .
\end{aligned}
$$

Hierbei wurde der Index a bei den $\mathrm{d} m_{k}$ fortgelassen, weil voraussetzungsgemäß nur Massenänderung durch Transport von der einen Phase zur anderen in Frage kommt:

8a Diese Autoren versuchen jedoch, Gl. (11) ,abzuleiten". Überhaupt wurde bisher der Definitionscharakter dieser oder ähnlicher Beziehungen selten erkannt. Daß Gl. (11) eine vernünftige Definition darstellt, erhellt z. B. das Folgende: Wird zu einem homogenen System eine infinitesimale Stoffmenge derselben Temperatur, desselben Druckes und derselben Zusammensetzung hinzugefügt, so gilt:

$$
\mathrm{d} U+\mathrm{d} V=\sum_{k=1}^{N} H_{k} \mathrm{~d}_{\mathrm{a}} m_{k},
$$

woraus mit Gl. (11) $\mathrm{d} Q=0$ folgt. 


$$
\mathrm{d} m^{\prime}{ }_{k}+\mathrm{d} m^{\prime \prime}{ }_{k}=0 .
$$

Wir nennen die Größe $\mathrm{d} m_{k}{ }^{\prime} / \mathrm{d} t=-\mathrm{d} m_{k}{ }^{\prime \prime} / \mathrm{d} t$ den „Massenstrom“ $J_{k}$ des Stoffes $k$ aus der Phase " in die Phase'. Entsprechend ist

$J_{Q} \equiv \frac{\mathrm{d}_{\mathrm{i}}{ }^{\prime} Q}{\mathrm{~d} t}=-\frac{\mathrm{d}_{\mathrm{i}}{ }^{\prime \prime} Q}{\mathrm{~d} t}+\sum_{k}\left(H_{k}{ }^{\prime \prime}-H_{k}{ }^{\prime}\right) J_{k}$

derWärmestrom von der Phase" zur Phase' (s.S.732). Die Größe $J_{Q}$ ist nur bei stationären Zuständen der Messung zugänglich: dann wird gemäß Gl. (15) $\mathrm{d}_{\mathrm{i}} Q=-\mathrm{d}_{\mathrm{a}} Q$. (Dies trifft auch noch für den ,,stationären Zustand zweiter Ordnung" $z u$, der für die prinzipielle Meßbarkeit der , U̇berführungswärmen“ wichtig ist ${ }^{3}$.)

Sämtliche sonst noch in der Literatur ${ }^{9,10}$ als ,Wärmestrom“ oder ,Energiestrom“ bezeichneten Größen $J^{\prime}{ }_{Q}$ sind von der allgemeinen Form

$$
J_{Q^{\prime}}=J_{Q}+\sum_{k=1}^{N} \gamma_{k} J_{k}
$$

\section{d) Kontinuierliche Systeme}

Wir betrachten ein Volumenelement eines kontinuierlichen Systems, d. h. eines Systems, in dem die intensiven Zustandsvariablen (Temperatur, Druck, Konzentrationen, chemische Potentiale usw.) von den Raumkoordinaten abhängen. Ein solches Volumenelement ist ein ,offenes System“.Wir berücksichtigen nun auch makroskopische kinetische Energie, die zweckmäßigerweise auf die Schwerpunktsgeschwindigkeit des Volumenelementes bezogen wird, und makroskopische potentielle Energie, die auf dem Vorhandensein äußerer Felder beruht. Diese Kraftfelder kennzeichnen wir durch die Vektoren $\vec{K}_{1}, \vec{K}_{2}, \ldots, \vec{K}_{N}$, wobei $\vec{K}_{k}$ die auf die Masseneinheit bezogene äußere Kraft ist, die auf die Teilchen des Stoffes $k$ wirkt. Es sei ferner $\varrho_{k}$ die Dichte des Stoffes $k, \varrho=\sum_{k=1}^{N} \varrho_{k}$ die Gesamtdichte, $\vec{v}_{k}$ die mittlere Geschwindigkeit der Teilchen des Stoffes $k$,

$$
\vec{v} \equiv \frac{1}{\varrho} \sum_{k=1}^{N} \varrho_{k} \vec{v}_{k}
$$

die Schwerpunktsgeschwindigkeit des Volumenelementes und

9 I. Prigogine, Étude thermodynamique des Phénomènes irréversibles, Dunod, Editeurs, Paris, und Editions Desoer, Lüttich, 1947.

$$
P=\left(\begin{array}{rrr}
p+p_{11} & p_{12} & p_{13} \\
p_{21} & p+p_{22} & p_{23} \\
p_{31} & p_{32} & p+p_{33}
\end{array}\right)
$$

der Drucktensor, der die Reibungsdrucke $p_{i j}=p_{j i}$ $(i, j=1,2,3)$ und den statischen Druck $p$ enthält. Der ,Diffusionsstrom“ “ $\vec{J}_{k}$, d. h. der Vektor der Diffusionsstromdichte des Stoffes $k$, ist durch die Gleichung

$$
\vec{J}_{k} \equiv \varrho_{k}\left(\vec{v}_{k}-\vec{v}\right) \quad(k=1,2, \ldots, N) .
$$

gegeben. $\tilde{U}$ sei die spezifische innere Energie.

Wir definieren nun den Vektor $\vec{W}$ der Wärmestromdichte, die wir kurz als Wärmestrom bezeichnen, durch folgende Beziehung:

$$
\begin{gathered}
\frac{\partial}{\partial t}\left(\varrho \tilde{U}+\frac{\varrho}{2} v^{2}\right)+\operatorname{div}\left(\varrho \tilde{U} \vec{v}+\frac{\varrho}{2} v^{2} \vec{v}\right) \\
-\sum_{k=1}^{N} \varrho_{k} \vec{K}_{k} \overrightarrow{v_{k}}+\operatorname{div}(P, \vec{v})+\operatorname{div}\left(\sum_{k=1}^{N} H_{k} \overrightarrow{J_{k}}\right) \\
\equiv-\operatorname{div} \vec{W} .
\end{gathered}
$$

Wenn wir uns auf den Standpunkt eines mit der Geschwindigkeit $\vec{v}$ bewegten Beobachters stellen, dann sind die drei ersten Terme der linken Seite die Zunahmegeschwindigkeit der (auf die Volumeneinheit bezogenen) inneren, kinetischen und potentiellen Energie des Volumenelementes, der vierte Term die pro Zeit- und Volumeneinheit gegen die Reibungsdrucke und den statischen Druck vom betrachteten Volumenelement geleistete Arbeit und der fünfte (letzte) Term der linken Seite das Analogon zu $-\sum_{k} H_{k} \frac{\mathrm{d}_{\mathrm{a}} m_{k}}{\mathrm{~d} t}$ in Gl. (11). Damit haben wir $-\operatorname{div} \vec{W}$ in Analogie zu dQ/d $t$ in Gl. (11) definiert.

Auch hier sind eine Reihe weiterer ,Wärmeströme“ oder „Energieströme“ in Gebrauch. Sie alle haben die Gestalt [vgl. Gl. (12) und (18)]

$$
\vec{W}^{\prime}=\vec{W}+\sum_{k=1}^{N} \gamma_{k} \vec{J}_{k}
$$

worin die $\gamma_{k}$ skalare Größen sind. Am meisten benutzt wird die Größe

$$
\vec{W} *=\vec{W}+\sum_{k=1}^{N} H_{k} \vec{J}_{k},
$$

${ }^{10} \mathrm{~S}$. R. De Groot, Thermodynamics of Irreversible Processes, North Holland Publishing Company, Amsterdam 1951. 
durch die man Gl. (19) rein formal abkürzen kann. Meixner ${ }^{11,12,13}$, der zuerst die Grundgleichungen der thermodynamisch-phänomenologischen Theorie der irreversiblen Prozesse in kontinuierlichen Systemen vollständig entwickelt hat, geht stets von der Größe $\vec{W}^{*}$ aus, die er ,Energiestrom“ nennt. Prigogine ${ }^{9}$ und De Groot ${ }^{10}$ bezeichnen $\vec{W}^{*}$ als „Wärmestrom“. Dadurch erklärt sich der Name „,reduzierter Wärmestrom" für die Größe $\vec{W}$. Kirkwood und Crawford ${ }^{14}$ wiederum führen den Vektor

$\vec{W}^{* *}=\vec{W}+\sum_{k=1}^{N} p V_{k} \vec{J}_{k}=\vec{W}^{*}-\sum_{k=1}^{N} U_{k} \vec{J}_{k}$

als ,Wärmestrom“ ein.

e) Gründe für unsere Wahl des Wärmestromes

Wir müssen nun noch die Gründe dafür auseinandersetzen, warum wir die Größen $\mathrm{d} Q / \mathrm{d} t, J_{Q}$ und $\vec{W}$, definiert durch die Gln. (11), (15) und (19) und bisher jeweils als ,,reduzierter Wärmestrom" bezeichnet, eingeführt haben.

Zunächst bietet die Benutzung dieser Größen eine Reihe spezieller Vorteile bei der thermodynamisch-phänomenologischen Behandlung der irreversiblen Prozesse. $\mathrm{Zu}$ diesen Vorteilen gehören: die einfache und vollständige Beschreibung der thermomagnetischen und galvanomagnetischen Effekte bei Verwendung des Wärmestroms $\vec{W}$ (Mazur und Prigogin $\mathrm{e}^{15}$ ), der enge Zusammenhang zwischen $J_{Q}$ bzw. $\vec{W}$ und den Eastman-Wagnerschen Überführungswärmen ${ }^{3}$, die besonders bei nichtisothermen Transportvorgängen in Elektrolyten eine große Rolle spielen ${ }^{16}$, usw.

Von den Gründen allgemeinerer Art, die unseren „Wärmestrom“ vor anders definierten Wärmeströmen auszeichnen, seien genannt:

I. Der Wärmestrom d $Q / \mathrm{d} t$ bzw. $J_{Q}$ bzw. $\vec{W}$ wird von einer Verschiebung der willkürlich festgelegten

11 J. Meixner, Ann. Physik (5) 39, 333 [1941]; 43, 244 [1943]; Z. physik. Chem., Abt. B 53, 235 [1943].

12 J. Meixner, Z. Naturforschg. 7 a, 553 [1952].

13 J. Meixner, Z. Naturforschg. 8a, 69 [1953].

${ }_{14}$ J. G. Kirkwood u. B. Crawford jr., J. physic. Chem. 56, 1048 [1952].

${ }_{15}$ P. Mazur u. I. Prigogine, J. Physique Radium 12, 616 [1951].

${ }^{16}$ R. Haase, Trans. Faraday Soc. 49, 724 [1953].
Nullpunkte der inneren Energien der einzelnen Stoffe nicht berührt, während z. B. der Strom $\vec{W}^{*}$, Gl. (21), vom Nullpunkt der Energiezählung abhängt. Da vom kinetischen Standpunkt eine zweckmäßige Verallgemeinerung des Begriffes ,Wärmestrom" auf offene Systeme so erfolgen sollte, daß die einem System zugeführte „Wärme“ die in das System transportierte kinetische Energie der ungeordneten Molekularbewegungen bedeutet, kommt von diesem Standpunkt aus wohl die Größe $\vec{W}$, nicht aber $\vec{W}^{*}$ als ,,Wärmestrom“ in Frage; denn offensichtlich enthält $\vec{W}^{*}$ noch mikroskopische potentielle Energie, deren Nullpunkt ja willkürlich festgelegt werden kann. Allerdings teilt der Wärmestrom $\vec{W}$ den Vorzug der Invarianz gegenüber Verschiebungen der Energienullpunkte noch mit anderen Wärmeströmen, z. B. mit der Größe $\vec{W}^{* *}$, Gl. (22). Daher genügt das angegebene Argument, das von Tolhoek und De Groot ${ }^{17}$ stammt, noch nicht zur eindeutigen Auszeichnung der Größe $\vec{W}$.

II. Eine plausible Form des Ausdrucks für die „Entropieströmung " 1,3 bei homogenen offenen bzw. kontinuierlichen Systemen ergibt sich nur bei Verwendung der Größe d $Q / \mathrm{d} t$ bzw. $\vec{W}$ als ,Wärmestrom" (Prigogine).

III. Wenn man, wie oft erforderlich, von der Schwerpunktsgeschwindigkeit auf eine andere Bezugsgeschwindigkeit umrechnen will ${ }^{3}$, ist der Ausdruck für die „Entropieerzeugung" bei mechanischem Gleichgewicht $(\mathrm{d} \vec{v} / \mathrm{d} t=0)$ invariant gegenüber dieser Transformation, wenn man als ,,Wärmestrom" die Größe $\vec{W}$ zugrunde legt (Prigogine).

IV. Wir betrachten den Vorgang der Wärmeleitung ohne chemische Reaktionen in einem kontinuierlichen System ${ }^{18}$. Wir nennen ,homogene Durchmischung " den Zustand, bei dem Druck- und Konzentrationsgradienten sowie äußere Kräfte ver-

Hierzu als Ergänzung: R. Ha a se, Z. Elektrochem. 57, 87, 448 [1953].

${ }_{17}$ H. A. Tolhoek u. S. R. De Groot, Physica 18, 780 [1952].

18 Diese Betrachtungen lassen sich sofort auf den Wärmeübergang zwischen zwei homogenen offenen Systemen (Abschnitt c) übertragen, wenn man $\vec{W}$ durch $J_{Q}$ und grad $T$ durch $T^{\prime}-T^{\prime \prime}$ ersetzt. Über Wärmeleitung in chemisch reagierenden Medien siehe nächstes Kapitel. 
schwinden. Ein ,,stationärer Zustand“ ist im betrachteten Falle durch die Bedingungen $\vec{J}_{k}=0$ $(k=1,2, \ldots, N)$ gekennzeichnet. Den Ausgangspunkt für die experimentelle Ermittlung einer „Wärmeleitfähigkeit" bildet eine ,Wärmeleitungsgleichung", d.h. eine Temperatur-Ort-Zeit-Beziehung. Wir werden daher im vorliegenden komplizierten Falle, bei dem Diffusion, Thermodiffusion usw. auftreten können, zwei ,Wärmeleitfähigkeiten" durch folgende Verabredung definieren: Der in der Wärmeleitungsgleichung im Term

$\operatorname{div}(\lambda \operatorname{grad} T)$ für $\operatorname{grad} p=0$,

$$
\left.\operatorname{grad}\left(\frac{\varrho_{k}}{\varrho}\right)=0, \vec{K}_{k}=0\right\}(k=1,2, \ldots, N)
$$

bzw. div $(\varkappa \operatorname{grad} T)$ für $\vec{J}_{k}=0$ )

auftretende Koeffizient $\lambda$ bzw. $x$ ist die ,Wärmeleitfähigkeit für homogene Durchmischung" bzw. die ,Wärmeleitfähigkeit für den stationären $\mathrm{Zu}$ stand".

Man kann nun folgendes zeigen ${ }^{1,3}$ :

A) Die Größe $\lambda$ findet sich in der Gleichung $\vec{W}=-\lambda \operatorname{grad} T$ (homogene Durchmischung)

wieder, nicht aber in der Beziehung

$\vec{W}^{\prime}=-\lambda^{\prime} \operatorname{grad} T$ (homogene Durchmischung),

wobei $\vec{W}^{\prime}$ gemäß Gl. (20) irgendein anderer ,,Wärmestrom" oder ,Energiestrom" und $\lambda$ ' eine von $\lambda$ verschiedene Größe ist.

B) Die Größe $\varkappa$ findet sich in der Relation

$$
\vec{W}=-\varkappa \operatorname{grad} T \text { (stationärer Zustand), }
$$

aber auch in jeder beliebigen Beziehung der Form

$$
\overrightarrow{W^{\prime}}=-\varkappa \operatorname{grad} T \text { (stationärer Zustand) }
$$

wieder, weil gemäß Gl. (20) für den stationären $\mathrm{Zu}$ stand $\left(\vec{J}_{k}=0\right)$ alle ,Wärmeströme“ zusammenfallen.

C) Aus dem positiv definiten Charakter der lokalen Entropieerzeugung läßt sich ableiten:

$$
\begin{gathered}
x>0, \\
\lambda-x>0
\end{gathered}
$$

und daher auch

$$
\lambda>0 .
$$

19 S. Chapman u. T. G. Cowling, The Mathematical Theory of Nonuniform Gases, Cambridge 1952.

${ }^{20}$ K. E. Grew u. T. L. I b b s, Thermal Diffusion in Gases, Cambridge 1952.
Für die durch Gl. (24) definierte Größe $\lambda^{\prime}$ läßt sich hingegen die Beziehung $\lambda^{\prime}>0$ nicht allgemein begründen.

Demnach ist es auch vom Standpunkt des Begriffs der „Wärmeleitfähigkeit“ zweckmäßig, den Vektor $\vec{W}$ als ,Wärmestrom“ zu benutzen.

Hat sich einmal die Erkenntnis durchgesetzt, daß ein ,Wärmestrom" nur durch Definition eingeführt werden kann und daß die Größen $\mathrm{d} Q / \mathrm{d} t, J_{Q}$ und $\vec{W}$ bei weitem die nützlichsten sind, so wird sich ein besonderer Name für diese Größen erübrigen. Einstweilen möchte der Verfasser aber zur Vermeidung von Mißverständnissen die Bezeichnung ,,reduzierter Wärmestrom“ für $\mathrm{d} Q / \mathrm{d} t, J_{Q}$ und $\vec{W}$ beibehalten, obwohl dieser Name kaum anders als ,,historisch“" $\mathrm{zu}$ rechtfertigen ist.

f) Ein Beispiel für den Vergleich mit der kinetischen Theorie

Wir sahen, daß der ,,reduzierte Wärmestrom" $\vec{W}$ infolge der Eigenschaft der Invarianz gegen Verschiebungen der Energienullpunkte zu derjenigen Klasse von ,Wärmeströmen" gehört, die auch vom kinetischen Standpunkt aus vernünftig sind. Wir wollen nun an einem einfachen Beispiel zeigen, daß die kinetische Theorie in konkreten Fällen gerade die Größe $\vec{W}$ als Wärmestrom definiert.

In der kinetischen Theorie der Transporterscheinungen, die von Enskog und Chapman für einatomige ideale Gase entwickelt worden ist, wird ein Wärmestrom $\vec{Q}$ als die pro Zeiteinheit durch die Flächeneinheit transportierte kinetische Energie der ungeordneten Bewegung der Moleküle definiert. Es ergibt sich für binäre Gasmischungen der Ausdruck ${ }^{19,20}$

$$
\vec{Q}=-\varkappa \operatorname{grad} T+c R T \frac{D_{\mathrm{T}}}{D}\left(\overrightarrow{v_{1}}-\overrightarrow{v_{2}}\right) .
$$

Hierin ist $D_{\mathrm{T}}$ der Thermodiffusionskoeffizient (mit entgegengesetzten Vorzeichen wie in ${ }^{3}$ ), $D$ der Diffusionskoeffizient, $R$ die Gaskonstante und $c$ die totale molare Volumenkonzentration. $\psi$ ist die Wärmeleitfähigkeit für den stationären Zustand $\left(\overrightarrow{v_{1}}=\overrightarrow{v_{2}}\right)$.

Andererseits ergibt die thermodynamisch-phänomenologische Theorie der irreversiblen Prozesse ${ }^{21}$ für beliebige binäre Gemische:

${ }^{21}$ Siehe $^{3}$, S. 148, Gln. $(5,148)$ und $(5,149)$. Zur Umrechnung von $\overrightarrow{J_{1}^{\prime}}$ auf $\overrightarrow{v_{1}}-\overrightarrow{v_{2}}$ siehe nächstes Kapitel, Gln. (33), (37) und (38). 
$\vec{W}=-\varkappa \operatorname{grad} T+\frac{D_{\mathrm{T}}}{D} x_{1}\left(\frac{\partial \bar{\mu}_{1}}{\partial x_{1}}\right)_{T, p} c\left(\overrightarrow{v_{1}}-\overrightarrow{v_{2}}\right)$,

worin $x_{1}$ der Molenbruch und $\bar{\mu}_{1}$ das molare chemische Potential des Stoffes 1 ist. Dieser Ausdruck ist vollkommen allgemeingültig, da er nur den $\mathrm{On}$ sagerschen Reziprozitätssatz enthält.

Setzt man

$$
\left(\frac{\partial \vec{\mu}_{1}}{\partial x_{1}}\right)_{T, p}=\frac{R T}{x_{1}}
$$

so geht Gl. (29) in Gl. (28) über. Wir sehen also, $\operatorname{da} \vec{Q}=\vec{W}$ ist.

Nebenbei kommen wir zu dem Schluß, daß Gl. (28) nicht nur für binäre ideale Mischungen aus einatomigen Gasen, sondern für alle (gasförmigen oder flüssigen) Mischungen gilt, bei denen Gl. (30) erfüllt ist, d. h. für beliebige binäre ideale Gemische und ideal verdünnte Lösungen. Diese Tatsache ist deshalb von Bedeutung, weil manchmal Gl. (28) als Ausgangspunkt für die allgemeine Behandlung des Diffusionsthermoeffektes vorausgesetzt wird, während die exakte Beziehung durch Gl. (29) gegeben ist (vgl. ${ }^{22}$ ).

\section{Wärmeleitfähigkeit in reagierenden} Zweistoffgemischen

Ein beliebiges fluides Medium enthalte die Substanz B, die durch eine Dissoziationsreaktion v Molekeln des Stoffes C pro Molekel B geben kann:

$$
\mathrm{B} \rightleftharpoons v \mathrm{C} .
$$

Der Index 1 bzw. 2 beziehe sich auf die Molekelsorte $\mathrm{B}$ bzw. C. Dann gilt für die Molmassen $M_{i}$ bzw. Molenbrüche $x_{i}$ :

bzw.

$$
M_{1}=v M_{2}
$$

$$
x_{1}=1-x_{2} .
$$

Die spezifische Reaktionsenthalpie $H_{\mathrm{R}}$ hängt mit der molaren Reaktionsenthalpie $\bar{H}_{\mathrm{R}}$ wegen Gl. (32) folgendermaßen zusammen $\left(\bar{H}_{i}=M_{i} H_{i}=\right.$ partielle molare Enthalpie):

$\bar{H}_{\mathrm{R}}=\bar{H}_{1}-v \bar{H}_{2}=M_{1}\left(H_{1}-H_{2}\right)=M_{1} H_{\mathrm{R}}$.

Die Bedingung für das lokale chemische Gleichgewicht lautet:

$$
\bar{\mu}_{1}-v \bar{\mu}_{2}=0, \operatorname{grad}\left(\bar{\mu}_{1}-v \bar{\mu}_{2}\right)=0,
$$

worin $\bar{\mu}_{i}$ das molare chemische Potential des Stoffes $i$ ist.

${ }^{22}$ R. Ha ase, Z. Elektrochem. 54, 450 [1950].
Die letzte Gleichung läßt sich auf Grund bekannter thermodynamischer Beziehungen auch schreiben:

$$
\frac{v x_{1}+x_{2}}{x_{2}}\left(\frac{\partial \bar{\mu}_{1}}{\partial x_{1}}\right)_{T, p} \operatorname{grad} x_{1}=\frac{\bar{H}_{R}}{T} \operatorname{grad} T,
$$

wobei verschwindender Druckgradient $(\operatorname{grad} p=0)$ vorausgesetzt ist.

Bezüglich der Transporterscheinungen ist unser System, in dem Konzentrations- und Temperaturgradienten bestehen, als Zweistoffgemisch zu behandeln. Wir führen als „Diffusionsstrom“ die Größe

$$
\vec{J}_{i}^{\prime} \equiv c_{i}\left(\vec{v}_{i}-\vec{u}\right),(i=1,2)
$$

mit

$$
\vec{u} \equiv x_{1} \overrightarrow{v_{1}}+x_{2} \overrightarrow{v_{2}}
$$

ein. Hierin ist $c_{i}=\varrho_{i} / M_{i}=x_{i} c$ die molare Volumenkonzentration des Stoffes $i\left(c=c_{1}+c_{2}\right)$.

Aus (37) und (38) folgt:

$$
\vec{J}_{1}^{\prime}+\vec{J}_{2}^{\prime}=0 .
$$

Es gilt für den Zusammenhang mit dem früher benutzten Diffusionsstrom:

$\vec{J}_{1}=-\vec{J}_{2}=\frac{M_{1} M_{2}}{M_{1} x_{1}+M_{2} x_{2}} \vec{J}_{1}^{\prime}=\frac{M_{1}}{v x_{1}+x_{2}} \vec{J}_{1}^{\prime}$.

Die konventionellen Gleichungen zur Beschreibung der Diffusion, Thermodiffusion, Wärmeleitung und des Diffusionsthermoeffektes lauten für $\operatorname{grad} p=0$ :

$$
\begin{gathered}
{\overrightarrow{J^{\prime}}}_{1}=-{\overrightarrow{J^{\prime}}}_{2}=-D c \operatorname{grad} x_{1}-\frac{D_{\mathrm{T}} c}{T} \operatorname{grad} T, \\
\vec{W}=-\beta \operatorname{grad} x_{1}-\lambda \operatorname{grad} T .
\end{gathered}
$$

Hierin ist $\vec{W}$ der ,,reduzierte Wärmestrom“, $D$ der Diffusionskoeffizient (für Diffusion der Molekeln B gegen die Molekeln C), $D_{\mathrm{T}}$ der Thermodiffusionskoeffizient, $\beta$ der Diffusionsthermokoeffizient (beide mit entgegengesetztem Vorzeichen wie in ${ }^{3} \mathrm{und}^{22}$ ) und $\lambda$ die Wärmeleitfähigkeit für homogene Durchmischung bei gehemmter Reaktion (siehe Abschn. 1.e).

Die thermodynamisch-phänomenologische Theorie der irreversiblen Prozesse ergibt als Konsequenz des Onsagerschen Reziprozitätssatzes die für ein beliebiges Zweistoffgemisch gültige Beziehung 3,22

$$
\beta=\frac{D_{\mathrm{T}} c}{x_{2}}\left(\frac{\partial \bar{\mu}_{1}}{\partial x_{1}}\right)_{T, p} .
$$

Aus den Gln. (41)-(43) folgt sofort der Ausdruck (29) und die explizite Relation für die Wärmeleit- 
fähigkeit $\varkappa$ für den stationären Zustand bei gehemmter Reaktion ${ }^{3,22}$ :

$$
\varkappa=\lambda-\frac{D_{T}^{2} c}{D T x_{2}}\left(\frac{\partial \bar{\mu}_{1}}{\partial x_{1}}\right)_{T, p},
$$

übrigens ein Beispiel für die Erfüllung der Ungleichung $(27 \mathrm{~b})$.

Wir wollen nun die Wärmeleitfähigkeit $\lambda_{\mathrm{A}}$ bei währendem Gleichgewicht berechnen ${ }^{23}$. Sie ist, entsprechend unseren Ausführungen auf S.735, dadurch definiert, daß sie in der Wärmeleitungsgleichung im Term

$$
\operatorname{div}\left(\lambda_{\mathrm{A}} \operatorname{grad} T\right) \text { für } \bar{\mu}_{1}-v \bar{\mu}_{2}=0
$$

auftritt. Nach Meixner ${ }^{12}$ findet sich die so definierte Größe $\lambda_{\mathrm{A}}$ in der Beziehung

$$
W^{*}=-\lambda_{\mathrm{A}} \operatorname{grad} T \text { (währendes Gleichgewicht) }
$$

wieder. Gemäß Gl. (21) und (34) können wir auch schreiben:

$$
\begin{gathered}
\vec{W}+H_{\mathrm{R}} \vec{J}_{1}=-\lambda_{\mathrm{A}} \operatorname{grad} T \\
\text { (währendes Gleichgewicht) }
\end{gathered}
$$

oder, gemäß Gl. (34) und Gl. (40),

$$
\vec{W}+\frac{\bar{H}_{\mathrm{R}}}{v x_{1}+x_{2}} \vec{J}_{1}^{\prime}=-\lambda_{\mathrm{A}} \operatorname{grad} T
$$

(währendes Gleichgewicht).

Tragen wir die Bedingung des lokalen chemischen Gleichgewichts, Gl. (36), die Transportgleichungen (41) und (42) sowie die Relation (43) in Gl. (45) ein, so erhalten wir den gewünschten Zusammenhang:

$$
\begin{aligned}
\lambda_{\mathrm{A}}=\lambda+\frac{x_{2} c \bar{H}_{\mathrm{R}}^{2} D}{\left(v x_{1}+x_{2}\right)^{2} T\left(\partial \bar{\mu}_{1} / \partial x_{1}\right) T, p} \\
\quad+\frac{2 c \bar{H}_{\mathrm{R}} D_{\mathrm{T}}}{\left(v x_{1}+x_{2}\right) T} .
\end{aligned}
$$

Gl. (46) ist einer von Meixner ${ }^{12}$ angegebenen Beziehung äquivalent. Für den speziellen Fall $v=1$ (Umlagerungsreaktion) geht Gl. (46) direkt in eine von Franck ${ }^{24}$ angegebene Gleichung über. Unsere Herleitung zeigt, wie sich das vorliegende Problem mit einem Minimum von Aufwand bewältigen läßt ${ }^{24 a}$.

\footnotetext{
${ }^{23}$ Der Index A zeigt an, daß die ,,Affinität“ $A=$ $\bar{\mu}_{1}-v \bar{\mu}_{2}$ konstant gleich Null gehalten wird.

${ }_{24}$ E. U. Franck, Z. physik. Chem. 201, 16 [1952], Gl. (18).

24a Inzwischen erhielt der Verf. Kenntnis von der Arbeit von I. Prigogine u. R. Buess, Bull. Acad. roy. Belgique (Cl. Sc.) 38, 711 [1952]. Diese Autoren erhalten ebenfalls Gl. (46), allerdings auch ohne Klärung des Begriffes ,Wärmeleitfähigkeit".
}

Wir wollen nun noch die Formel von Nernst ${ }^{25}$ für die Wärmeleitfähigkeit eines dissoziierenden idealen Gases zur obigen Gleichung in Beziehung setzen. Aus der bisherigen Literatur ist dieser $\mathrm{Zu}$ sammenhang nicht klar ersichtlich.

Bei Abwesenheit chemischer Reaktionen nimmt Nernst als Stationaritätsbedingung für ein binäres ideales Gasgemisch in einem Temperaturgefälle (grad $T \neq 0)$ die Beziehung $\left(p_{1}=p x_{1}=\right.$ Partialdruck)

$$
\operatorname{grad} p_{1}=0
$$

an, die mit $\operatorname{grad} p=0$ auf die Bedingung

$$
\operatorname{grad} x_{1}=0
$$

führt. Heute beschreiben wir den stationären $\mathrm{Zu}$ stand durch Gl. (41) mit $\vec{J}_{1}^{\prime}=0$. Daher entspricht Gl. (47) der Ignorierung der Thermodiffusion $\left(D_{\mathrm{T}}=0\right)^{26}$.

Bei Ablauf der chemischen Reaktion im Temperaturgefälle denkt sich Nernst den Partialdruckgradienten dadurch gegeben, da $B$ sich an jedem Orte das zu der betreffenden Temperatur gehörige Reaktionsgleichgewicht einstellt. Dies entspricht unserer lokalen Gleichgewichtsbedingung (36), wenn wir sie auf ideale Gase spezialisieren. Nernst behandelt dann an Hand des Beispiels $\mathrm{N}_{2} \mathrm{O}_{4} \rightleftharpoons 2 \mathrm{NO}_{2}$ den Spezialfall $v=2$. Er findet die Formel

$$
\lambda_{\mathrm{A}}=\lambda+\frac{\alpha(1-\alpha) p \bar{H}_{\mathrm{R}}^{2} D^{\prime}}{(1+\alpha) R^{2} T^{3}} .
$$

Hierin ist $\alpha$ der Dissoziationsgrad und $D^{\prime}$ der ,,Diffusionskoeffizient", den Nernst durch die Beziehung

$$
\begin{gathered}
c_{1} \vec{v}_{1}=-\frac{D^{\prime}}{R T} \operatorname{grad} p_{1}=-D^{\prime} c \operatorname{grad} x_{1} \\
\text { (stationärer Zustand) }
\end{gathered}
$$

definiert.

Wir wollen nun zeigen, wie Gl. (46) bei idealen Gasen für $v=2$ und bei Vernachlässigung der Thermodiffusion auf Gl. (48) führt.

Für den Dissoziationsgrad $\alpha$ gilt:

${ }^{25}$ W. Nernst, Festschrift Ludwig Boltzmann, Joh. Ambr. Barth, Leipzig 1904, S. 904.

${ }^{26}$ Selbstverständlich würde Gl. (47) auch auf eine ,Entmischung" führen, wenn man die Konzentration nicht durch $x_{1}$, sondern durch $c_{1}$ mißt. Dies hat jedoch mit Thermodiffusion nichts zu tun. Vgl. hierzu De Groot ${ }^{27}$.

${ }^{27}$ S. R. De Groot, L'effet Soret, Diss. Amsterdam 1945. 


$$
x_{1}=\frac{1-\alpha}{1+(v-1) \alpha}, x_{2}=\frac{v \alpha}{1+(v-1) \alpha} .
$$

Wir betrachten den Sonderfall

$$
\begin{array}{r}
\left(\frac{\partial \bar{\mu}_{1}}{\partial x_{1}}\right)_{T, p}=\frac{R T}{x_{1}}, p=c R T \text { (ideale Gase), } \\
D_{\mathrm{T}}=0 \text { (keine Thermodiffusion) }
\end{array}
$$

und finden hierfür aus Gl. (46) mit Gl. (50) :

$\lambda_{\mathrm{A}}=\lambda+\frac{x_{1} x_{2} p \bar{H}_{\mathrm{R}}^{2} D}{\left(v x_{1}+x_{2}\right)^{2} R^{2} T^{3}}=\lambda+\frac{\alpha(1-\alpha) p \bar{H}_{\mathrm{R}}^{2} D}{v R^{2} T^{3}}$.

Um den Zusammenhang zwischen $D$ und $D^{\prime}$ zu gewinnen, schreiben wir die Massenbilanz an:

$$
\begin{aligned}
& \frac{\partial c_{1}}{\partial t}=-\operatorname{div}\left(c_{1} \overrightarrow{v_{1}}\right)+w, \\
& \frac{\partial c_{2}}{\partial t}=-\operatorname{div}\left(c_{2} \overrightarrow{v_{2}}\right)-v w,
\end{aligned}
$$

worin die Reaktionsgeschwindigkeit $w$ die durch die chemische Reaktion bedingte Zunahmegeschwindigkeit der Konzentration des Stoffes 1 im betrachteten Volumenelement ist. Im stationären Zustande $\left(\partial c_{i} / \partial t=0, i=1,2\right)$ gilt also:

$$
v c_{1} \vec{v}_{1}+c_{2} \overrightarrow{v_{2}}=0 \text { (stationärer Zustand). }
$$

Hieraus resultiert für die Schwerpunktsgeschwindigkeit $\vec{v}=\left(\varrho_{1} / \varrho\right) \overrightarrow{v_{1}}+\left(\varrho_{2} / \varrho\right) \overrightarrow{v_{2}}$ bzw. die mittlere molare Geschwindigkeit $\vec{u}$, Gl. (38), mit Gl. (32):

$$
\vec{v}=0, \vec{u} \neq 0 \text { (stationärer Zustand). }
$$

Mit Gl. (40) folgt aus Gl. (49) und Gl. (54) für den nicht-stationären Fall:

$$
\begin{aligned}
M_{1} c_{1}\left(\overrightarrow{v_{1}}-\vec{v}\right)=\vec{J}_{1} & =\frac{M_{1}}{v x_{1}+x_{2}} \vec{J}_{1}^{\prime} \\
& =-M_{1} D^{\prime} c \operatorname{grad} x_{1} .
\end{aligned}
$$

Hieraus ergibt sich durch Vergleich mit Gl. (41) für $D_{\mathrm{T}}=0$ :

$$
D=D^{\prime}\left(v x_{1}+x_{2}\right) \text {. }
$$

Durch Eintragen von Gl. (55) in Gl. (53) erhalten wir für $v=2$ bei Berücksichtigung von Gl. (50) die Nernstsche Formel (48).

\section{Zum Prinzip der minimalen Entropie- erzeugung}

Irreversible Prozesse führen häufig zu einem ,stationären Zustand", d. h. zu einem Nichtgleichgewichtszustand, bei dem die Variablen, die das Sy- stem makroskopisch beschreiben, nicht mehr von der Zeit abhängen. Es liegt nahe zu fragen, ob und unter welchen Bedingungen die allgemeine charakteristische Größe der irreversiblen Prozesse, die „Entropieerzeugung“, bei Annäherung an den stationären Zustand einen Extremwert annimmt.

Diese Frage ist zunächst von Prig ogine für spezielle Fälle, dann von De Groot und anderen allgemeiner untersucht worden. Bei allen diesen Rechnungen sind drei Voraussetzungen gemacht worden :

1. Die Geschwindigkeiten der irreversiblen Prozesse werden durch die linearen ,,phänomenologischen Ansätze“ beschrieben.

2. Die phänomenologischen Koeffizienten (für welche die Onsagerschen Reziprozitätsbeziehungen gelten) sind zeitlich konstant.

3. Dem betrachteten System werden bestimmte Nebenbedingungen auferlegt, die von Fall zu Fall verschieden sein können und deren physikalische Realisierbarkeit eines Kommentars bedarf.

Es ist bekannt, daß die phänomenologischen Ansätze für Transportprozesse gute Näherungen sind, da sie z. B. auf das Ohmsche, Fouriersche und Ficksche Gesetz bzw. die entsprechenden verallgemeinerten linearen Beziehungen führen. Im Falle der chemischen Reaktionen ist jedoch der Gültigkeitsbereich der linearen Relationen zwischen Reaktionsgeschwindigkeiten und Affinitäten sehr klein, wie man beim Vergleich mit den experimentell gesicherten kinetischen Ansätzen sieht.

Die Annahme der zeitlichen Konstanz der phänomenologischen Koeffizienten hingegen bildet keine so einschneidende Voraussetzung mehr, wenn man einmal die Gültigkeit der linearen Ansätze anerkannt hat. Dies sieht man am schnellsten an Hand eines Beispiels ein. In einem geschlossenen Gefäß, das mit einer beliebigen homogenen Mischung gefüllt ist, herrsche ein Temperaturgefälle, das dadurch erzeugt wird, daß die Begrenzungsflächen des Systems auf verschiedenen (konstanten) Temperaturen gehalten werden. Dadurch setzt Thermodiffusion und nach einiger Zeit gewöhnliche Diffusion ein, bis ein stationärer Zustand mit zeitlich konstanter Konzentrations- und Temperaturverteilung erreicht ist. Wenn auch der Diffusionskoeffizient, der Thermodiffusionskoeffizient und die Wärmeleitfähigkeit, die in eindeutigem Zusammenhang mit den in den phänomenologischen Ansätzen be- 
nutzten Koeffizienten stehen, im allgemeinen von der Temperatur und der Konzentration abhängen, kann man doch die zeitliche Änderung ihrer Mittelwerte vernachlässigen, da die Mittelwerte der Temperatur und der Konzentration sich praktisch nicht ändern. Bei der mathematischen Beschreibung eines solchen Trennvorganges macht man sogar meist die noch einschneidendere Voraussetzung, daß die Temperaturverteilung während des Prozesses der Annäherung an den stationären Endzustand als vorgegeben angesehen werden darf.

Die Diskussion der unter 3. genannten Voraussetzung kann nur bei einer näheren Betrachtung der Einzelfälle erfolgen, der wir uns jetzt zuwenden.

In homogenen offenen Systemen sind stationäre Zustände dadurch möglich, daß die durch chemische Reaktionen im Inneren des Systems erzeugten oder verbrauchten Stoffe mit einer solchen Geschwindigkeit durch die Begrenzungen des Systems hinausoder hineinströmen, daß alle Konzentrationen konstant bleiben. Es läßt sich zeigen ${ }^{2}$, daß nur unter ganz bestimmten Voraussetzungen die Bedingungen der Konstanz der Affinitäten mehrerer Reaktionen realisierbar sind, die nach Prigogine ${ }^{9,28}$ und De Groot $^{10} \mathrm{zu}$ einem Zusammenfallen des stationären Zustands mit dem der minimalen Entropieerzeugung führen. Denbigh ${ }^{29}$ hat an einem einfachen Beispiel gezeigt, wie sehr die Allgemeingültigkeit des Prinzips der minimalen Entropieerzeugung bei chemischen Reaktionen durch die Forderung der Gleichgewichtsnähe, impliziert durch die phänomenologischen Ansätze, eingeschränkt wird. Es muß also vor zu weitgehenden Analogieschlüssen bei der Anwendung des Minimumprinzips auf biologische Vorgänge ${ }^{30,31}$ gewarnt werden: die Bedingungen, unter denen dieses Prinzip bei chemischen Reaktionen gilt, dürfen nicht aus dem Auge verloren werden ${ }^{3,32}$.

Diskontinuierliche Systeme sind im einfachsten Falle geschlossene Systeme, die sich aus zwei homogenen offenen Systemen zusammensetzen. Hier ist eine allgemeine Formulierung des Prinzips der mini-

${ }^{28}$ I. Prigogine, Bull. Acad. roy. Belgique (Cl. Sc.) 31, 600 [1945].

${ }^{29}$ K. G. Den bigh, Trans. Faraday Soc.48,389 [1952].

${ }^{30}$ I. Prigogine u. J. M. Wiame, Experientia [Basel] 2, 451 [1946].

${ }^{31}$ L. von Bertalanffy, Science [New York] 111,23 [1950].

32 R. Haa se, Z. Elektrochem. 55, 566 [1951].

${ }^{33}$ I. Prigogine u. R. Defay, Bull. Acad. roy. Belgique (Cl. Sc.) 32, 694 [1946]; 33, 48 [1947]. malen Entropieerzeugung dann möglich, wenn die physikalisch realisierbaren Nebenbedingungen der Konstanz einer oder mehrerer generalisierter „Kräfte" entsprechen. Daher fallen die von Prigogine und Defay ${ }^{33}$ angegebenen Sätze (für die irreversible Verdampfung und Kondensation in isothermen Zweistoffsystemen) aus dem Rahmen der verallgemeinerungsfähigen Theoreme heraus. Der Befund von Prigogine ${ }^{9}$ für den gekoppelten Stoff- und Wärmeübergang zwischen zwei homogenen Einstoff- oder Zweistoffsystemen hingegen erweist sich als Spezialfall des allgemeinen Theorems ${ }^{2}$ : ,,Während des Ablaufs beliebiger Transportprozesse in geschlossenen Systemen nimmt bei gegebener Temperaturverteilung die Entropieerzeugung genau dann einen positiven Minimalwert an, wenn ein stationärer Nichtgleichgewichtszustand erreicht ist." Die Nebenbedingung der gegebenen Temperaturverteilung ist hier einfach mit der Konstanz der Temperaturen der beiden Teilsysteme identisch. Das Theorem gilt auch noch, wenn man neben dem Stoff- und Wärmetransport eine chemische Reaktion in Gleichgewichtsnähe zuläßt² .

Bei kontinuierlichen Systemen gibt es zwei Möglichkeiten, die Nebenbedingungen festzulegen. Im ersten Falle denkt man sich die Temperatur überall vorgegeben, wie es schon Prigogine ${ }^{9}$, De Groot ${ }^{10}$ und der Verfasser ${ }^{22}$ bei der Thermodiffusion angenommen haben (vgl. das oben genannte Beispiel). Dann ergibt sich, wie der Verfasser ${ }^{2}$ allgemein zeigen konnte, wieder das obige Theorem ${ }^{34}$. Im zweiten Falle denkt man sich die Temperatur und die chemischen Potentiąle der einzelnen Stoffe an den Begrenzungsflächen des Systems vorgegeben, wie es später Wergeland ${ }^{35}$ und Mazur ${ }^{36}$ angenommen haben, und betrachtet nicht die lokale Entropieerzeugung, sondern die gesamte Entropieerzeugung des Systems. In diesem Falle, der hinsichtlich der Bedingungen für die Temperatur realistischer als der vorige ist, hat man sich das System als offen vorzustellen, damit die Konzentrationen an den Systemgrenzen konstant gehalten werden können.

${ }^{34}$ Bei dem allgemeinen Beweis dieses Theorems für kontinuierliche Systeme hätte der Verfasser ${ }^{2}$ von vornherein die chemischen Reaktionen ausschließen müssen, da sonst die gewählte Nebenbedingung allzu künstlich ist.

${ }^{35} \mathrm{H}$. Wergeland, Kgl. norske Vidensk. Selsk., Forh. 24, 110 [1951].

${ }^{36}$ P. Mazur, Bull. Acad. roy. Belgique (Cl. Sc.) 38, 182 [1952]. 
Dann läßt sich das Minimumprinzip auf die reine Wärmeleitung, die elektrische Leitung und das gleichzeitige Auftreten von Transportphänomenen und chemischen Reaktionen ausdehnen ${ }^{36}$. Auch die Stabilität dieser stationären Zustände minimaler Entropieerzeugung läßt sich nachweisen ${ }^{36}$. Bezüg- lich der chemischen Reaktionen gelten die gleichen Einschränkungen wie oben.

Herrn Prof. J. Meixner danke ich für wertvolle Diskussionen und Herrn Prof. E. Jenckel dafür, daß er die Durchführung dieser Arbeit in seinem Institut ermöglicht hat.

\title{
Spinortheorie der Elementarteilchen I
}

\author{
(Grundlagen der modifizierten Theorie)
}

\section{Von Ferdinand Cap}

Aus dem Institut für theoretische Physik der Universität Innsbruck

(Z. Naturforschg. 8a, 740-744 [1953]; eingegangen am 4. September 1953)

\begin{abstract}
Während die übliche Spinortheorie der Elementarteilchen mit mehr als $2(2 s+1)$ Spinorkomponenten arbeitet und Spinoren mit punktierten und unpunktierten Indices verwendet werden, begnügt sich die hier vorgeschlagene Theorie mit $2(2 s+1)$ Spinorkomponenten, herrührend von Spinoren, die nur eine Indexsorte besitzen und Differentialgleichungen 2. Ordnung genügen. Es wird zunächst über die übliche Theorie referiert und der Grundgedanke der neuen Theorie wird herausgearbeitet.
\end{abstract}

$B^{\text {etrat }}$ etrachtet man die heutige Theorie der Elementarteilchen ${ }^{1}$, so kann man sich des Eindrucks nicht erwehren, daß die Anzahl der Komponenten der Wellenfunktion, die für die Beschreibung eines bestimmten Teilchens herangezogen wird, nicht übereinstimmt mit den physikalischen Möglichkeiten eines Elementarteilchens. Es ist doch so, daß ein bestimmtes Elementarteilchen zwei Freiheitsgrade besitzt: Ladung und Spin. Die Ladung kann die Zustände positiv elektrisch und negativ elektrisch annehmen; der Spinfreiheitsgrad hat beim Spin $s$ bekanntlich die Einstellmöglichkeitęn $s, s-1, s-2$, $\ldots,-(s-2),-(s-1),-s$, also insgesamt $2 s+1$ Möglichkeiten. Zusammen mit den durch den Ladungsfreiheitsgrad gegebenen zwei Möglichkeiten sind dies $2(2 s+1)$ durch Messung voneinander scharf zu unterscheidende physikalische Zustände (Tab. 1).

Beim Leptonfeld beispielsweise kann man ein Elektron mit ,,aufwärts" und eines mit ,,abwärts" gerichtetem Spin, ein Positron mit ,,aufwärts“ und eines mit ,abwärts" gerichtetem Spin unterscheiden. Es hat sich nun aus Gründen der einfachen Rechenweise in der Physik eingebürgert, einen Vorgang mit $z$ physikalisch voneinander unterscheidbaren Freiheitsgraden durch $z$-komponentige mathe-

${ }^{1}$ G. Wentzel, Einführung in die Quantentheorie der Wellenfelder, Deuticke-Verlag, Wien 1943; L. de B rogli e, Théorie Générale des Particules à Spin, Gauthier-Villars-Verlag, Paris 1943.

\begin{tabular}{|l|c|c|}
\hline \multicolumn{1}{|c|}{$\begin{array}{c}\text { Name } \\
\text { des Teilchens }\end{array}$} & Spin $s$ & $\begin{array}{c}\text { Zahl } \\
\text { der Zustände }\end{array}$ \\
\hline skalares Meson & 0 & 2 \\
Lepton $^{2}$ & $1 / 2$ & 4 \\
vektorielles Meson & 1 & 6 \\
3/2-Teilchen & $3 / 2$ & 8 \\
Graviton & 2 & 10 \\
$5 / 2$ & $5 / 2$ & 12 \\
3 & 3 & 14 \\
\hline
\end{tabular}

Tab. 1.

matische Größen zu beschreiben. So wird z. B. die Bewegung eines starren Körpers, die bekanntlich 6 Freiheitsgrade besitzt, durch 6 Größen - Geschwindigkeitsvektor der Schwerpunktstranslation und Winkelgeschwindigkeit um den Schwerpunkt - dargestellt. Weniger Komponenten würden den Vorgang nicht vollkommen beschreiben - mehr Komponenten wären formal einführbar, physikalisch aber sinnlos und würden zusätzliche Nebenbedingungen erfordern (mit deren Hilfe die überflüssigen Komponenten eliminiert werden könnten).

Die heutige Theorie der Elementarteilchen arbeitet aber nach einem solchen Schema - es werden weitaus mehr Komponenten verwendet als physi-

${ }^{2}$ F. Cap, Acta physica austriaca, 6, 35 [1952], s. dort Zitate aus Physic. Rev. 